\title{
Effect of MMSE Channel Estimation on BER Performance of Orthogonal Space-Time Block Codes in Rayleigh Fading Channels
}

\author{
Lennert Jacobs, Student Member, IEEE, and Marc Moeneclaey, Fellow, IEEE
}

\begin{abstract}
We present a simple closed-form expression for the BER of orthogonal space-time block codes (OSTBCs) with MMSE channel estimation, assuming PAM and QAM constellations and flat Rayleigh block fading. The BER expression is exact for a certain class of OSTBCs, and provides a very accurate approximation for other OSTBCs.
\end{abstract}

Index Terms-Bit error rate (BER), channel estimation, Rayleigh fading, multiple-input multiple-output (MIMO), orthogonal space-time block codes (OSTBCs).

\section{INTRODUCTION}

$\mathbf{I}$ $\mathrm{N}$ order to enable reliable communication over rapidly time-varying channels, multiple-input multiple-output (MIMO) wireless communication systems exploit spatial diversity by using multiple transmit and/or receive antennas. A maximum diversity order of $N_{\mathrm{t}} N_{\mathrm{r}}$ can be achieved (with $N_{\mathrm{t}}$ and $N_{\mathrm{r}}$ denoting the number of transmit and receive antennas, respectively), provided that proper spacetime coding is used. Orthogonal space-time block codes (OSTBCs) [1]-[3] are considered a very attractive transmit diversity technique, since they achieve full spatial diversity, and the maximum-likelihood decoding algorithm reduces to symbol-by-symbol detection, based only on linear processing at the receiver. Owing to the simple detection algorithm, bit error rate (BER) expressions for OSTBCs are easily derived when perfect channel knowledge (PCK) is assumed (e.g. [4]-[7]).

In practice, however, the channel state information (CSI) is not a priori known and errors resulting from imperfect channel estimation degrade the system performance. In [8], the effect of channel estimation errors on the BER of spacetime block codes was demonstrated by means of simulations. In [9] and [10], the symbol error rate (SER) of OSTBCs in presence of imperfect channel estimation was examined. High-SNR expressions for the pairwise error probability (PEP) were derived under quite general conditions in [11], using an eigenvalue approach. In [12], an exact closed-form expression for the PEP of both orthogonal and non-orthogonal spacetime codes in the case of least-squares channel estimation was obtained by means of characteristic functions. In a fading

Paper approved by S. A. Jafar, the Editor for Wireless Communication Theory and CDMA of the IEEE Communications Society. Manuscript received September 11, 2007; revised February 14, 2008.

The authors wish to acknowledge the activity of the Network of Excellence in Wireless COMmunications NEWCOM++ of the European Commission (contract no. 216715) that motivated this work.). The first author also gratefully acknowledges the support from the Fund for Scientific Research in Flanders (FWO-Vlaanderen).

The authors are with the Department of Telecommunications and Information Processing, Ghent University, Gent B-9000, Belgium (e-mail: \{Lennert.Jacobs, Marc.Moeneclaey\}@telin.ugent.be).

Digital Object Identifier 10.1109/TCOMM.2009.05.070455 environment, however, the BER upper bound resulting from the PEP does not converge to the true BER at high SNR. Therefore, in this paper we assess the true BER of OSTBCs. For OSTBCs satisfying a particular condition, we derive a simple closed-form expression for the exact BER in case of pulse amplitude modulation (PAM) and quadrature amplitude modulation (QAM) signal constellations. For OSTBCs that do not satisfy this condition, we point out that the BER expression provides a very accurate approximation.

Throughout this paper, the superscript $H$ denotes the conjugate matrix transpose. $\operatorname{tr}(\mathbf{X})$ and $\|\mathbf{X}\|$ refer to the trace and the Frobenius norm of $\mathbf{X}$, respectively.

\section{SIGNAL MODEL}

We consider a MIMO communication system with $N_{\mathrm{t}}$ transmit antennas and $N_{\mathrm{r}}$ receive antennas, and a flat Rayleigh fading propagation channel. Transmission is organized in frames: in one frame, each transmit antenna sends $K_{\mathrm{p}}$ known pilot symbols and $K$ coded data symbols. The pilot symbols are used by the receiver to estimate the channel, which is assumed to be constant within one frame of $N_{\mathrm{fr}}=K+K_{\mathrm{p}}$ symbols and changes independently from one frame to another (block fading). The $N_{\mathrm{r}} \times N_{\text {fr }}$ received signal matrix $\mathbf{R}_{\text {tot }}$ is given by

$$
\mathbf{R}_{\text {tot }}=\left[\mathbf{R}_{\mathrm{p}}, \mathbf{R}\right]=\mathbf{H}\left[\mathbf{A}_{\mathrm{p}}, \mathbf{A}\right]+\mathbf{W}
$$

where the $N_{\mathrm{t}} \times K_{\mathrm{p}}$ pilot matrix $\mathbf{A}_{\mathrm{p}}$ and the $N_{\mathrm{t}} \times K$ data matrix $\mathbf{A}$ consist of the pilot symbols and the coded data symbols, respectively, transmitted at each transmit antenna. The propagation channel is represented by the $N_{\mathrm{r}} \times N_{\mathrm{t}}$ complex random matrix $\mathbf{H}$, whose elements are independent identically distributed (i.i.d.) zero-mean circularly symmetric complex Gaussian (ZMCSCG) random variables with unit variance (i.e., each channel coefficient has independent real and imaginary parts with zero mean and variance $1 / 2$ ). The $N_{\mathrm{r}} \times N_{\text {fr }}$ matrix $\mathbf{W}$ represents additive spatially and temporally white noise and consists of i.i.d. ZMCSCG random variables with variance $N_{0}$.

An orthogonal space-time block code transforms a vector $\mathbf{s}(k)$ of $N_{\mathrm{s}}$ information symbols $s_{i}(k), 1 \leq i \leq N_{\mathrm{s}}$, into a $N_{\mathrm{t}} \times K_{\mathrm{c}}$ coded symbol matrix $\mathbf{C}(k)$, with $k$ denoting the block index. Assuming that $K$ is a multiple of $K_{\mathrm{c}}$, the transmitted data symbol matrix is given by $\mathbf{A}=$ $\sqrt{E_{\mathrm{S}}}\left[\mathbf{C}(1), \cdots, \mathbf{C}\left(K / K_{\mathrm{c}}\right)\right]$. In this paper, we restrict our attention to OSTBCs from complex linear processing orthogonal designs [1], such that the entries of the symbol matrices are linear combinations of the information symbols and their complex conjugate. Hence, the code matrix $\mathbf{C}$ can be written 
as (we drop the index $k$ for notational convenience)

$$
\mathbf{C}=\sum_{i=1}^{N_{\mathrm{s}}}\left(\mathbf{C}_{i} s_{i}+\mathbf{C}_{i}^{\prime} s_{i}^{*}\right),
$$

where the $N_{\mathrm{t}} \times K_{\mathrm{c}}$ matrices $\mathbf{C}_{i}$ and $\mathbf{C}_{i}^{\prime}$ comprise the coefficients of $s_{i}$ and $s_{i}^{*}$ in the matrix $\mathbf{C}$, respectively. Without loss of generality, we may assume that the matrices $\mathbf{C}$ are scaled in such way that they satisfy the following orthogonality condition:

$$
\mathbf{C C}^{H}=\left(\lambda \sum_{i=1}^{N_{\mathrm{s}}}\left|s_{i}\right|^{2}\right) \mathbf{I}_{N_{\mathrm{t}}},
$$

where $\lambda=K_{\mathrm{c}} / N_{\mathrm{s}}$ and $\mathbf{I}_{N_{\mathrm{t}}}$ is the $N_{\mathrm{t}} \times N_{\mathrm{t}}$ identity matrix. Considering a normalized information symbol constellation $\left(\mathbb{E}\left[\left|s_{i}\right|^{2}\right]=1\right)$, it follows from (3) that the average energy of the transmitted coded symbols is given by $E_{\mathrm{s}}$. Similarly, the average energy of the pilot symbols is given by $E_{\mathrm{p}}$.

\section{Pilot-Based Channel Estimation}

The receiver can estimate the channel $\mathbf{H}$ using $\mathbf{R}_{\mathrm{p}}$ and the known pilot matrix $\mathbf{A}_{\mathrm{p}}$. Assuming orthogonal training sequences, i.e., the matrix $\mathbf{A}_{\mathrm{p}}$ has orthogonal rows such that $\mathbf{A}_{\mathrm{p}} \mathbf{A}_{\mathrm{p}}^{H}=K_{\mathrm{p}} E_{\mathrm{p}} \mathbf{I}_{N_{\mathrm{t}}}$, the linear minimum mean-square error (MMSE) channel estimate [13] is given by

$$
\hat{\mathbf{H}}=\frac{1}{N_{0}+K_{\mathrm{p}} E_{\mathrm{p}}} \mathbf{R}_{\mathrm{p}} \mathbf{A}_{\mathrm{p}}^{H} .
$$

Defining the channel estimation error as $\hat{\boldsymbol{\Delta}}=\mathbf{H}-\hat{\mathbf{H}}$, the following properties can be derived:

- $\hat{\mathbf{H}}$ and $\hat{\boldsymbol{\Delta}}$ are statistically independent Gaussian random matrices;

- The components of $\hat{\mathbf{H}}$ are independent and identically distributed (i.i.d.) ZMCSCG random variables with variance $\sigma_{\hat{\mathbf{H}}}^{2}=K_{\mathrm{p}} E_{\mathrm{p}} /\left(N_{0}+K_{\mathrm{p}} E_{\mathrm{p}}\right)$;

- The components of $\hat{\boldsymbol{\Delta}}$ are i.i.d. ZMCSCG random variables with variance $\sigma_{\hat{\boldsymbol{\Delta}}}^{2}=N_{0} /\left(N_{0}+K_{\mathrm{p}} E_{\mathrm{p}}\right)$.

Allocating a large total energy $K_{\mathrm{p}} E_{\mathrm{p}}$ to pilot symbols yields an accurate channel estimate, but on the other hand gives rise to a reduction of the symbol energy $E_{\mathrm{s}}$. When $E_{\mathrm{b}}$ denotes the energy per information bit and $\gamma \triangleq E_{\mathrm{p}} / E_{\mathrm{s}}$ is the ratio of the pilot energy to the data energy, we have

$$
E_{\mathrm{s}}=\frac{K}{K+\gamma K_{\mathrm{p}}} \rho \log _{2}(M) E_{\mathrm{b}},
$$

where $\rho=N_{\mathrm{s}} /\left(N_{\mathrm{t}} K_{\mathrm{c}}\right)$ and $M$ denote the code rate and the number of constellation points, respectively. Hence, $E_{\mathrm{s}}$ decreases when the number of pilot symbols $K_{\mathrm{p}}$ is increased.

\section{BIt ERror RATE ANALYSIS}

We consider a mismatched receiver that uses the estimated channel matrix $\hat{\mathbf{H}}$ in the same way a maximum-likelihood (ML) receiver would use the actual channel matrix $\mathbf{H}$. In this way, the detection algorithm reduces to symbol-by-symbol detection. Denoting by $\mathbf{R}^{\prime}$ the received signal matrix corresponding to the transmitted symbol matrix $\mathbf{C}$, the decision variable $u_{i}$ related to the symbol $s_{i}, 1 \leq i \leq N_{\mathrm{s}}$, is given by

$$
u_{i}=\frac{\operatorname{tr}\left(\mathbf{C}_{i}^{H} \hat{\mathbf{H}}^{H} \mathbf{R}^{\prime}+\mathbf{R}^{\prime H} \hat{\mathbf{H}} \mathbf{C}_{i}^{\prime}\right)}{\lambda \sqrt{E_{\mathrm{s}}}\|\hat{\mathbf{H}}\|^{2}} .
$$

Expanding (6) yields $u_{i}=s_{i}+n_{i}, 1 \leq i \leq N_{\mathrm{s}}$, where the disturbance term $n_{i}$ contains contributions from the channel noise and the channel estimation error $\hat{\boldsymbol{\Delta}}$, and is Gaussian when conditioned on $\mathbf{C}$.

\section{A. M-PAM constellation}

We obtain the BER from the conditional BER of the $i$ th information symbol (conditioned on the estimated channel matrix and the transmitted information symbol vector $\mathbf{s}=$ $\left.\left(s_{1}, \cdots, s_{N_{\mathrm{s}}}\right)\right)$ :

$$
\begin{aligned}
& \operatorname{BER}_{M-\mathrm{PAM}}=\mathbb{E}_{i, \mathbf{s}, \hat{\mathbf{H}}}\left[\sum_{b \in \Psi} \frac{N\left(s_{i}, b\right)}{\log _{2} M} P_{i}(\mathbf{s}, b, \hat{\mathbf{H}})\right] \\
& =\frac{1}{N_{\mathrm{s}}} \frac{1}{M^{N_{\mathrm{s}}}} \sum_{i=1}^{N_{\mathrm{s}}} \sum_{\mathbf{s} \in \Psi^{N_{\mathrm{s}}}} \sum_{b \in \Psi} \frac{N\left(s_{i}, b\right)}{\log _{2} M} \mathbb{E}_{\hat{\mathbf{H}}}\left[P_{i}(\mathbf{s}, b, \hat{\mathbf{H}})\right] .
\end{aligned}
$$

In (7), $\Psi$ denotes the normalized $M$-PAM constellation, $N\left(s_{i}, b\right)$ represents the Hamming distance between the binary representations of the transmitted symbol $s_{i}$ and the decoded symbol $b$, and $P_{i}(\mathbf{s}, b, \hat{\mathbf{H}})$ is the probability that $u_{i}$ is located in the decision area of the constellation point $b$ (when the transmitted symbol vector and the channel estimate are known). When $d_{1}\left(s_{i}, b\right)$ and $d_{2}\left(s_{i}, b\right)$ denote the distances between the transmitted constellation point $s_{i}$ and the boundaries of the decision area of $b$, with $d_{1}\left(s_{i}, b\right)<d_{2}\left(s_{i}, b\right)$ (we set $d_{2}\left(s_{i}, b\right)=\infty$ if $b$ is an outer constellation point), the latter probability is given by

$$
P_{i}(\mathbf{s}, b, \hat{\mathbf{H}})=Q\left(\frac{d_{1}\left(s_{i}, b\right)}{\sigma_{\mathrm{R}_{i}}(\mathbf{s}, \hat{\mathbf{H}})}\right)-Q\left(\frac{d_{2}\left(s_{i}, b\right)}{\sigma_{\mathrm{R}_{i}}(\mathbf{s}, \hat{\mathbf{H}})}\right),
$$

where $\sigma_{\mathrm{R}_{i}}(\mathbf{s}, \mathbf{H})$ denotes the standard deviation of the real part of $u_{i}$ and $Q($.$) is the complementary cumulative distribution$ function of a zero-mean Gaussian random variable with unit variance. Let the $N_{\mathrm{t}} \times N_{\mathrm{t}}$ matrix $\mathbf{C}_{\mathrm{R}_{i}}(\mathbf{s}), 1 \leq i \leq N_{\mathrm{s}}$, be defined as

$$
\mathbf{C}_{\mathrm{R}_{i}}(\mathbf{s}) \triangleq \mathbf{C}\left(\mathbf{C}_{i}+\mathbf{C}_{i}^{\prime}\right)^{H}
$$

which is a function of the information symbol vector $\mathbf{s}$ through the code matrix $\mathbf{C}$ (see (2)). It can be shown that

$$
\begin{aligned}
& \sigma_{\mathrm{R}_{i}}^{2}(\mathbf{s}, \hat{\mathbf{H}}) \\
& \quad=\frac{N_{0}}{2 \lambda E_{\mathrm{s}}\|\hat{\mathbf{H}}\|^{2}}\left(1+\frac{\sigma_{\hat{\mathbf{\Delta}}}^{2}}{N_{0}} \frac{E_{\mathrm{s}}\left\|\hat{\mathbf{H}} \mathbf{C}_{\mathrm{R}_{i}}(\mathbf{s})^{H}\right\|^{2}}{\lambda\|\hat{\mathbf{H}}\|^{2}}\right) .
\end{aligned}
$$

Since the expression between parentheses in (10) depends on $\hat{\mathbf{H}}$, the expectation of (8) over $\hat{\mathbf{H}}$ is difficult to compute. However, this dependence is removed if the considered OSTBC satisfies the following criterion for $1 \leq i \leq N_{\mathrm{s}}$ :

$$
\mathbf{C}_{\mathrm{R}_{i}}(\mathbf{s})^{H} \mathbf{C}_{\mathrm{R}_{i}}(\mathbf{s})=\beta_{\mathrm{R}_{i}}(\mathbf{s}) \mathbf{I}_{N_{\mathrm{t}}},
$$


where $\beta_{\mathrm{R}_{i}}(\mathbf{s})=\left\|\mathbf{C}_{\mathrm{R}_{i}}(\mathbf{s})\right\|^{2} / N_{\mathrm{t}}$. For OSTBCs satisfying (11), the variance (10) simplifies to

$$
\sigma_{\mathrm{R}_{i}}^{2}(\mathbf{s}, \hat{\mathbf{H}})=\frac{N_{0}}{2 \lambda E_{\mathrm{s}}\|\hat{\mathbf{H}}\|^{2}}\left(1+\frac{\sigma_{\hat{\mathbf{\Delta}}}^{2}}{N_{0}} \frac{E_{\mathrm{s}} \beta_{\mathrm{R}_{i}}(\mathbf{s})}{\lambda}\right),
$$

and the averaged Q-functions in (8) reduce to [14]

$$
\begin{aligned}
\mathbb{E}_{\hat{\mathbf{H}}}\left[Q\left(\frac{d_{j}\left(s_{i}, b\right)}{\sigma_{\mathrm{R}_{i}}(\mathbf{a}, \hat{\mathbf{H}})}\right)\right] & \\
= & \Omega\left(\frac{2 \lambda d_{j}^{2}\left(s_{i}, b\right)}{1+\frac{\beta_{\mathrm{R}_{i}}(\mathbf{s})}{\lambda \gamma K_{\mathrm{p}}}+\frac{1}{\gamma K_{\mathrm{p}}} \frac{N_{0}}{E_{\mathrm{s}}}} \frac{E_{\mathrm{s}}}{N_{0}}\right),
\end{aligned}
$$

for $j \in\{1,2\}$. In (13), the function $\Omega(\theta)$ is defined as

$$
\Omega(\theta) \triangleq\left[\frac{1-\mu}{2}\right]^{L} \sum_{k=0}^{L-1}\left(\begin{array}{c}
L-1+k \\
k
\end{array}\right)\left[\frac{1+\mu}{2}\right]^{k}
$$

where $L=N_{\mathrm{r}} N_{\mathrm{t}}$ denotes the spatial diversity achieved by the OSTBC, and $\mu=\sqrt{\theta /(2+\theta)}$.

Taking (5) into account, the ratio of the BER of the mismatched receiver to the BER with PCK $\left(K_{\mathrm{p}}=0\right)$ is easily obtained (at high $E_{\mathrm{b}} / N_{0}$ ):

$$
\begin{aligned}
& \frac{\mathrm{BER}_{M-\mathrm{PAM}, \mathrm{MMSE}}}{\mathrm{BER}_{M-\mathrm{PAM}, \mathrm{PCK}}} \\
& \quad=\left(1+\frac{\gamma K_{\mathrm{p}}}{K}\right)^{L} \frac{\mathbb{E}_{i, \mathbf{s}}\left[\left(1+\frac{\beta_{\mathrm{R}_{i}}(\mathbf{s})}{\lambda \gamma K_{\mathrm{p}}}\right)^{L} \xi\left(s_{i}\right)\right]}{\mathbb{E}_{s}[\xi(s)]},
\end{aligned}
$$

where the function $\xi(s)$ is defined as

$$
\xi(s) \triangleq \sum_{b \in \Psi} N(s, b)\left(\frac{1}{d_{1}^{2 L}(s, b)}-\frac{1}{d_{2}^{2 L}(s, b)}\right) .
$$

Given that the ratio (15) is not a function of $E_{\mathrm{b}} / N_{0}$, it follows that the PCK receiver and the mismatched receiver achieve the same diversity order, as observed in [13].

For OSTBCs that do not satisfy criterion (11), replacing the variance (10) by its average over the entries of $\hat{\mathbf{H}}$, when conditioned on $\|\hat{\mathbf{H}}\|^{2}$, also results in (12). In this way, (13) and (15) provide simple approximations of the BER and the BER degradation of the mismatched receiver.

\section{B. M-QAM constellation}

Now we consider square $M$-QAM information symbols with Gray mapping, which is equivalent to $\sqrt{M}$-PAM mapping for both the in-phase and quadrature information bits. In this way, the BER for QAM transmission reduces to the average of the BERs for the in-phase and quadrature information bits. For the sake of conciseness we provide only the QAM equivalent of criterion (11); the computation of the BER and the associated BER degradation follows the same lines as for PAM. Defining the matrix $\mathbf{C}_{\mathrm{I}_{i}}(\mathbf{s}) \triangleq \mathbf{C}\left(\mathbf{C}_{i}-\mathbf{C}_{i}^{\prime}\right)^{H}$, the exact BER for both the in-phase and quadrature bits can be computed analytically if the OSTBC satisfies the following criterion for $1 \leq i \leq N_{\mathrm{s}}$ :

$$
\left\{\begin{array}{l}
\mathbf{C}_{\mathrm{R}_{i}}(\mathbf{s})^{H} \mathbf{C}_{\mathrm{R}_{i}}(\mathbf{s})=\frac{\left\|\mathbf{C}_{\mathrm{R}_{i}}(\mathbf{s})\right\|^{2}}{N_{\mathrm{t}}} \mathbf{I}_{N_{\mathrm{t}}} \\
\mathbf{C}_{\mathrm{I}_{i}}(\mathbf{s})^{H} \mathbf{C}_{\mathrm{I}_{i}}(\mathbf{s})=\frac{\left\|\mathbf{C}_{\mathrm{I}_{i}}(\mathbf{s})\right\|^{2}}{N_{\mathrm{t}}} \mathbf{I}_{N_{\mathrm{t}}}
\end{array},\right.
$$

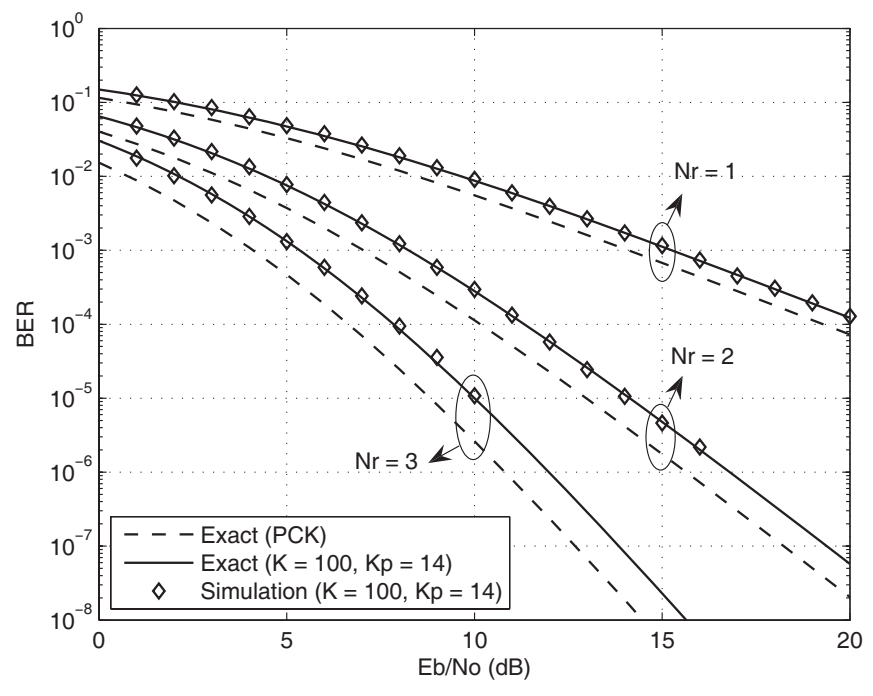

Fig. 1. BER of Alamouti's code, 4-QAM.

where $\mathbf{C}_{\mathrm{R}_{i}}(\mathbf{s})$ is given by (9). Examples of OSTBCs that satisfy (17) are given in [15], [1, eq. (38),(40)] and [2, eq. (62)]. For OSTBCs not satisfying (17), the resulting BER expression is a simple approximation of the actual BER.

\section{NUMERICAL RESUlts}

Alamouti's code [15] $\left(N_{\mathrm{t}}=K_{\mathrm{c}}=N_{\mathrm{s}}=2\right)$, is given by

$$
\mathcal{C}^{(2 \times 2)}=\left(\begin{array}{cc}
s_{1} & -s_{2}^{*} \\
s_{2} & s_{1}^{*}
\end{array}\right),
$$

and satisfies criterion (17), with $\left\|\mathbf{C}_{\mathrm{R}_{i}}(\mathbf{s})\right\|^{2}=\left\|\mathbf{C}_{\mathrm{I}_{i}}(\mathbf{s})\right\|^{2}=$ $N_{\mathrm{t}}\left(\left|s_{1}\right|^{2}+\left|s_{2}\right|^{2}\right)$ for $i=1,2$. Hence, the exact BER curves for PAM and QAM constellations can be derived analytically. Fig. 1 shows the exact BER curves for 4-QAM transmission employing Alamouti's code, for both the mismatched receiver and the PCK receiver (we assume $E_{\mathrm{p}}=E_{\mathrm{s}}$ ); also shown are computer simulation results for the mismatched receiver that confirm the analytical result. The corresponding BER degradation amounts to $1.15 \mathrm{~dB}$, irrespective of $N_{\mathrm{r}}$.

The OSTBC given by [1, eq. (39)] $\left(N_{\mathrm{t}}=3, K_{\mathrm{c}}=\right.$ $4, N_{\mathrm{s}}=3$ ), does not satisfy criterion (17). Fig. 2 illustrates the BER for a 16-QAM constellation, resulting from the PCK receiver (exact result) and the mismatched receiver (analytical approximation and simulation result). The simulations indicate that the approximation for the mismatched receiver is very accurate. The BER degradation amounts to $1.04 \mathrm{~dB}, 1.06 \mathrm{~dB}$ or $1.09 \mathrm{~dB}$ when $N_{r}$ equals 1,2 or 3 , respectively.

\section{CONCLUSIONS}

In this contribution, we investigated the BER performance of orthogonal space-time block codes under the assumption of pilot-based MMSE channel estimation at the receiver. The MIMO propagation channel was assumed to be affected by uncorrelated flat Rayleigh block fading.

For a class of OSTBCs, specified by a proposed criterion, we derived simple closed-form expressions of the exact BER and the related BER degradation for both PAM and QAM symbol constellations. These expressions depend on the number 


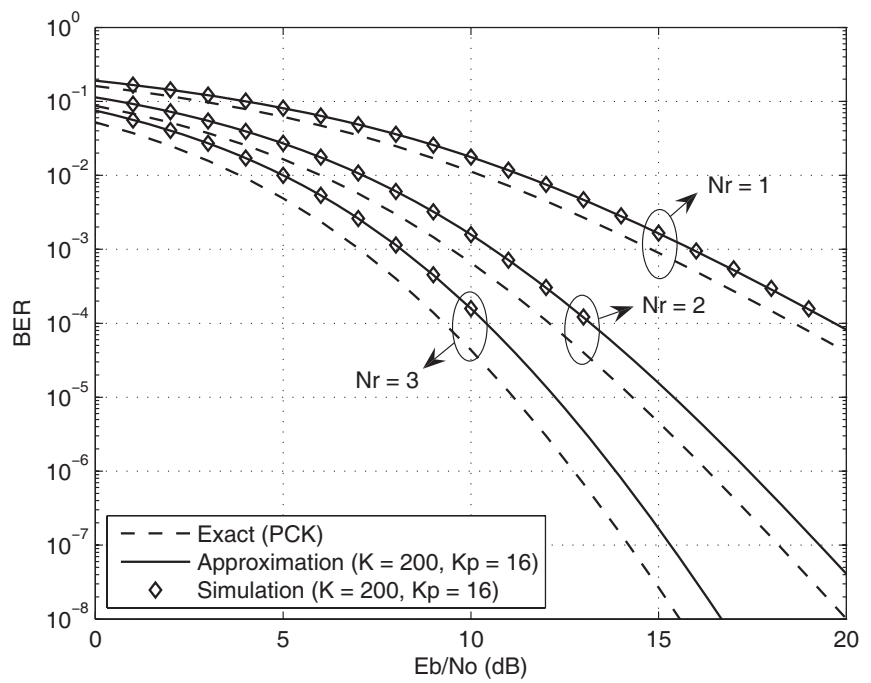

Fig. 2. BER of OSTBC given by [1, eq. (39)], 16-QAM.

of pilot symbols, data symbols, transmit antennas and receive antennas, on the considered constellation and on some specific properties of the OSTBC.

For OSTBCs not belonging to this specific class, we derived simple approximations of the BER and the BER degradation caused by channel estimation errors. According to simulations, however, these approximations turn out to be very accurate.

\section{REFERENCES}

[1] V. Tarokh, H. Jafarkhani, and A. Calderbank, "Space-time block codes from orthogonal designs," IEEE Trans. Inform. Theory, vol. 45, no. 5, pp. 1456-1467, July 1999.

[2] X.-B. Liang, "Orthogonal designs with maximal rates," IEEE Trans. Inform. Theory, vol. 49, no. 10, pp. 2468-2503, Oct. 2003.
[3] K. Lu, S. Fu, and X.-G. Xia, "Closed-form designs of complex orthogonal space-time block codes of rates $(k+1) /(2 k)$ for $2 k-1$ or $2 k$ transmit antennas," IEEE Trans. Inform. Theory, vol. 51, no. 12, pp. 4340-4347, Dec. 2005.

[4] S. Chennakeshu and J. Anderson, "Error rates for Rayleigh fading multichannel reception of MPSK signals," IEEE Trans. Commun., vol. 43, no. 234, pp. 338-346, Feb./Mar./Apr. 1995.

[5] I.-M. Kim and V. Tarokh, "Variable-rate space-time block codes in Mary PSK systems," IEEE J. Select. Areas Commun., vol. 21, no. 3, pp. 362-373, Apr. 2003.

[6] S.-H. Kim, I.-S. Kang, and J.-S. No, "Exact bit error probability of orthogonal space-time block codes with QAM," in Proc. IEEE Int. Symp. on Information Theory (ISIT), Yokohama, Japan, June 2003, p. 63.

[7] I.-M. Kim, "Exact BER analysis of OSTBCs in spatially correlated MIMO channels," IEEE Trans. Commun., vol. 54, no. 8, pp. 1365-1373, Aug. 2006.

[8] D. Mavares and R. Torres, "Channel estimation error effects on the performance of STB codes in flat frequency Rayleigh channels," in Proc. IEEE Vehicular Technology Conf. (VTC-Fall), Orlando, USA, Oct. 2003, pp. 647-651, vol. 1.

[9] E. Ko, C. Kang, and D. Hong, "Effect of imperfect channel information on M-QAM SER performance of orthogonal space-time block codes," in Proc. IEEE Vehicular Technology Conf. (VTC-Spring), Jeju Island, Korea, Apr. 2003, pp. 722-726, vol. 1.

[10] L. Tao, L. Jianfeng, H. Jianjun, and Y. Guangxin, "Performance analysis for orthogonal space-time block codes in the absence of perfect channel state information," in Proc. IEEE Int. Symp. Personal, Indoor and Mobile Radio Communications (PIMRC), Beijing, China, Sept. 2003, pp. 1012-1016 vol. 2.

[11] M. Brehler and M. Varanasi, "Asymptotic error probability analysis of quadratic receivers in Rayleigh-fading channels with applications to a unified analysis of coherent and noncoherent space-time receivers," IEEE Trans. Inform. Theory, vol. 47, no. 6, pp. 2383-2399, Sept. 2001.

[12] P. Garg, R. Mallik, and H. Gupta, "Performance analysis of spacetime coding with imperfect channel estimation," IEEE Trans. Wireless Commun., vol. 4, no. 1, pp. 257-265, Jan. 2005.

[13] G. Taricco and E. Biglieri, "Space-time decoding with imperfect channel estimation," IEEE Trans. Wireless Commun., vol. 4, no. 4, pp. 1874 1888, July 2005.

[14] J. Proakis, Digital Communications, 4th ed. McGraw-Hill, 2001.

[15] S. Alamouti, "A simple transmit diversity technique for wireless communications," IEEE J. Select. Areas Commun., vol. 16, pp. 1459-1478, Oct. 1998. 